\title{
Ultrasound-assisted dispersive liquid-liquid microextraction of tetracycline drugs from egg supplements before flow injection analysis coupled to a liquid waveguide capillary cell
}

\author{
Michael Pérez Rodríguez ${ }^{1}$ Helena Redigolo Pezza ${ }^{1} \cdot$ Leonardo Pezza $^{1}$
}

Received: 19 May 2016 / Revised: 15 June 2016 / Accepted: 20 June 2016 / Published online: 5 July 2016

(C) Springer-Verlag Berlin Heidelberg 2016

\begin{abstract}
A simple, rapid, and efficient ultrasound-assisted dispersive liquid-liquid microextraction (US-DLLME) method was developed for extraction of tetracycline residues from egg supplement samples, with subsequent determination by flow injection analysis (FIA) coupled to a liquid waveguide capillary cell (LWCC) and a controlled temperature heating bath. Tetracyclines react with diazotized p-sulfanilic acid, in a slightly alkaline medium, to form azo compounds that can be measured at $435 \mathrm{~nm}$. The reaction sensitivity improved substantially (5.12-fold) using an in-line heating temperature of $45^{\circ} \mathrm{C}$. Multivariate methodology was used to optimize the factors affecting the extraction efficiency, considering the volumes of extraction and disperser solvents, sonication time, extraction time, and centrifugation time. Good linearity in the range $30-600 \mu \mathrm{g} \mathrm{L}^{-1}$ was obtained for all the tetracyclines, with regression coefficients $(r)$ higher than 0.9974 . The limits of detection ranged from 6.4 to $11.1 \mu \mathrm{g} \mathrm{L}^{-1}$, and the recoveries were in the range 85.7-96.4\%, with relative standard deviation lower than $9.8 \%$. Analyte recovery was improved by approximately $6 \%$ when the microextraction was assisted by ultrasound. The results obtained with the proposed USDLLME-FIA method were confirmed by a reference HPLC method and showed that the egg supplement samples analyzed were suitable for human consumption.
\end{abstract}

Electronic supplementary material The online version of this article (doi:10.1007/s00216-016-9732-4) contains supplementary material, which is available to authorized users.

Leonardo Pezza

pezza@iq.unesp.br

1 Institute of Chemistry, State University of São Paulo-UNESP, R. Prof. Francisco Degni 55, P.O. Box 355, 14800-900 Araraquara, São Paulo, Brazil
Keywords Ultrasound-assisted dispersive liquid-liquid microextraction (US-DLLME) · Tetracyclines · Egg supplements $\cdot$ Flow injection analysis (FIA) $\cdot$ Liquid waveguide capillary cell (LWCC)

\section{Introduction}

Tetracycline (TC), oxytetracycline (OTC), chlortetracycline (CTC), and doxycycline (DC) constitute a class of broadspectrum antibacterial drugs that are widely used in agriculture for treating and preventing infectious diseases, or as growth promoters $[1,2]$. Tetracycline antibiotics are poorly metabolized in animals and unsafe levels of drug residues can therefore be present in foods such as meat, fish, honey, milk, and eggs, as well as in products derived from them [3, 4]. Eggs are an inexpensive animal-derived food that is readily available and has high nutritional value [5], and the presence of contaminants is not permitted.

The presence of tetracycline drugs in this type of foodstuff may represent a potential risk to the consumer because these compounds can cause allergic reactions, liver damage, and gastrointestinal disturbances, or induce pathogen resistance to antimicrobial agents [5-7].

In order to ensure human food safety, several international organizations have established maximum residue levels (MRLs) of veterinary drugs in foodstuffs from animal origins. For instance, in the case of eggs and the products derived from them, the Canadian [8] and Codex Alimentarius [9] norms have adopted MRLs of $400 \mu \mathrm{g} \mathrm{kg}^{-1}$ for tetracycline (TC), oxytetracycline (OTC), and chlortetracycline (CTC), while the European Union [10] sets a limit of $200 \mu \mathrm{g} \mathrm{kg}^{-1}$ for this compound class. Levels of tetracyclines in eggs that exceed the MRLs disqualify the product. Despite the existence of these MRLs, indiscriminate or improper uses of veterinary 
drugs in poultry farming can lead to problems of contamination. Hence, there is a need for the development of simple and reliable methods for the analysis of tetracyclines in eggs and in products derived from them.

The analytical techniques reported in the literature for the monitoring and determination of tetracycline antibiotics and other veterinary drugs in eggs are mostly chromatographic, including high performance liquid chromatography (HPLC) with ultraviolet (UV) [7, 11-13], fluorescence [14], or mass spectrometry (MS/MS) [1-4, 15-20] detection, and ultrahigh-pressure liquid chromatography-tandem mass spectrometry (UHPL-MS/MS) [5, 6].

The extraction of tetracyclines from egg samples prior to use of the above methods involves tedious and timeconsuming procedures that can be expensive and generally require larger volumes of organic solvents, compared to microextraction techniques. The most important are solvent extraction [5, 16], liquid-liquid extraction (LLE) [3], solidphase extraction (SPE) [1, 2, 4, 5, 11, 14, 15, 17, 19], ultrasonic-assisted extraction (UAE) [2], and pressurized liquid extraction (PLE) $[6,12]$. Furthermore, SPE modifications using molecularly imprinted solid-phase extraction (MISPE) $[13,20]$ and magnetic molecularly imprinted polymer solidphase extraction (MIP-SPE) [18] have also been used as extraction methods. However, the traditional extraction techniques must be miniaturized in order to comply with the principles of green chemistry [21].

Dispersive liquid-liquid microextraction (DLLME) is a novel and powerful preconcentration technique based on miniaturization of LLE, which uses a ternary solvent system [22]. In this procedure, an appropriate mixture of extraction and disperser solvents is rapidly injected into the aqueous sample to form a cloudy solution and the analytes in the aqueous sample are rapidly extracted into the fine droplets of the extraction solvent. The cloudy mixture is separated by centrifugation and the sedimented phase is collected with a microsyringe for subsequent analysis by chromatography or spectrometry $[7,23,24]$.

Ease of operation, low cost, high recovery, very short extraction time, and high enrichment factors are some of the advantages of DLLME. This technique has been widely used to determine different emerging pollutants [23-26] and inorganic species [27-29] in environmental water samples, and a method based on HPLC coupled to ionic liquid dispersive liquid-liquid microextraction (IL-DLLME) has been developed for the determination of tetracycline drugs in eggs [7].

There are few reports in the literature concerning the coupling of DLLME with flow injection analysis (FIA). Both procedures provide simplicity, rapidity, cost economy, good precision, and low consumption of reagents and solvents [23, $24,30,31]$. In combination, they offer an environmentally safer technique for the analysis of tetracyclines in eggs and in products derived from eggs.
In the present study, an ultrasonic-assisted dispersive liquid-liquid microextraction (US-DLLME) method was developed for the extraction of tetracycline antibiotics from egg supplement samples prior to spectrophotometric flow injection analysis using a liquid waveguide capillary cell (LWCC). The proposed method is based on a diazo coupling reaction suggested in the literature [31], which occurs between tetracyclines and diazotized p-sulfanilic acid in a slightly alkaline medium. The reaction product is then measured at $435 \mathrm{~nm}$. The proposed FIA procedure includes an in-line heating step to improve the sensitivity of the reaction. Multivariate methodologies were used to investigate and optimize the experimental parameters of the microextraction and flow injection system. The proposed US-DLLME-FIA method was validated under the best conditions using egg-based protein supplement samples.

\section{Experimental}

\section{Reagents and chemicals}

All solutions were prepared by dissolving analytical grade reagents in deionized water $\left(18.0 \mathrm{M} \Omega \mathrm{cm}\right.$ at $\left.25^{\circ} \mathrm{C}\right)$ obtained from a Milli-Q purification system (Millipore Corp., Bedford, MA, USA). Concentrated hydrochloric acid (37\% purity), psulfanilic acid ( $99 \%$ purity), sodium nitrite ( $99 \%$ purity), and trichloroacetic acid ( $99 \%$ purity) were obtained from Merck. Sodium acetate ( $99 \%$ purity) and standards of tetracycline hydrochloride ( $99 \%$ purity), chlortetracycline hydrochloride (98\% purity), oxytetracycline hydrochloride (99\% purity), and doxycycline hyclate ( $98 \%$ purity) were purchased from Sigma-Aldrich. HPLC-grade methanol, ethanol, acetone, and acetonitrile were obtained from Tedia. The extraction solvents chloroform (99\%), dichloromethane (99\%), and carbon tetrachloride (99\%) were purchased from Merck.

Working standard solutions of each tetracycline (TC, OTC, CTC, and DC) were prepared at concentrations from 30 to $600 \mu \mathrm{g} \mathrm{L}^{-1}$ by diluting the stock solutions with deionized water in the presence of sodium acetate $\left(0.02 \mathrm{~mol} \mathrm{~L}^{-1}\right)$. An alkaline $5.0 \times 10^{-2} \mathrm{~mol} \mathrm{~L}^{-1}$ working solution of sodium acetate was prepared by dissolving an appropriate amount of solid drug in deionized water in a $250-\mathrm{mL}$ volumetric flask. The diazotized p-sulfanilic acid solution (chromogenic reagent) was obtained using an aqueous mixture of p-sulfanilic acid and hydrochloric acid at concentrations of $1.2 \times 10^{-4}$ and $2.0 \times 10^{-4} \mathrm{~mol} \mathrm{~L}^{-1}$, respectively, plus sodium nitrite at a concentration of $2.4 \times 10^{-4} \mathrm{~mol} \mathrm{~L}^{-1}$.

\section{Apparatus}

The merging zones flow injection system (Fig. 1) employed a variable speed (1-50 rpm) six-channel peristaltic pump 




Fig. 1 Flow injection manifold used for the determination of tetracycline drugs. $P P$, peristaltic pump; $C$, carrier solution $\left(5.0 \times 10^{-2} \mathrm{~mol} \mathrm{~L}^{-1}\right.$ sodium acetate); $I C$, injector-commutator with sampling $(P l)$ and injection $(P 2)$ positions; $S L$, sampling loop (80 cm length, $402 \mu \mathrm{L}$ internal volume); $S$, sample; $R L$, reagent loop (120 cm length, $603 \mu \mathrm{L}$ internal volume); $R$, chromogenic reagent $\left(1.2 \times 10^{-4} \mathrm{~mol} \mathrm{~L}^{-1}\right.$ diazotized p-sulfanilic acid); $X$, confluence; $R C$, reaction coil (300 cm length); $T B$, thermostatic bath with temperature control $\left(45^{\circ} \mathrm{C}\right) ; D$, detector coupled to a liquid waveguide capillary cell (LWCC, $1.0 \mathrm{~m}$ path length, $550 \mu \mathrm{m}$ inner diameter, $250 \mu \mathrm{L}$ inner volume); $W$, waste

(Gilson Minipuls 3, Villiers-le-Bel, France) equipped with Tygon tubing (1.65 $\mathrm{mm}$ i.d.) for fluid propulsion. The FIA manifold also included a manual acrylic injector-commutator, an acrylic confluence, connectors, and polytetrafluoroethylene tubes $(0.8 \mathrm{~mm}$ i.d) for construction of the sample loop $(402 \mu \mathrm{L}$ internal volume, $80 \mathrm{~cm}$ length), reagent loop ( $603 \mu \mathrm{L}$ internal volume, $120 \mathrm{~cm}$ length), carrier lines, and reaction coil (200 cm length). An MA126 thermostatic bath equipped with a PT100 temperature sensor $\left(0.1^{\circ} \mathrm{C}\right.$ resolution $)$ and an LDS digital temperature controller was used to heat the reaction coil. The auxiliary sonication step of the extraction process was performed using an ultrasonic water bath (Branson $3210 \mathrm{R}-\mathrm{DTH}$, with $40 \mathrm{kHz}$ frequency and maximum power of $700 \mathrm{~W}$, the internal dimension: $304.8 \times 152.8 \times 150 \mathrm{~mm}$ i.d.). Maximum absorbance at $435 \mathrm{~nm}$ and $45^{\circ} \mathrm{C}$ was measured using a USB 4000 spectrophotometer (Ocean Optics, Dunedin, FL, USA) coupled to an LWCC 3100 liquid waveguide capillary cell (World Precision Instruments, Sarasota, FL, USA) with a $1.0 \mathrm{~m}$ path length $(550 \mu \mathrm{m}$ inner diameter and $250 \mu \mathrm{L}$ inner volume), and to a light source (Model LS-1LL halogen lamp, Ocean Optics), using two optical fiber cables with diameters of 600 and $200 \mu \mathrm{m}$, respectively.

The HPLC analyses were performed using a Shimadzu Prominence instrument equipped with an LC-20AT quaternary gradient pump, a SIL-20A HT autosampler, an SPD-M20A diode array detector, a CTO-20A column oven, and a CBM20A software interface. Chromatographic separation was achieved with an OmniSpher $\mathrm{C} 18$ reversed phase column $(250 \times 4.6 \mathrm{~mm}$ i.d., $5 \mu \mathrm{m})$, and the injection volume was $20 \mu \mathrm{L}$. The mobile phase consisted of $1.0 \times 10^{-2} \mathrm{~mol} \mathrm{~L}^{-1}$ oxalic acid (solvent $\mathrm{A}$ ) and acetonitrile (solvent $\mathrm{B}$ ), using the following gradient elution: $0-2.0 \mathrm{~min}(12-20 \% \mathrm{~B}) ; 2.0$ $4.0 \mathrm{~min}(20-27 \% \mathrm{~B}) ; 4.0-10.0 \mathrm{~min}(27-30 \% \mathrm{~B})$; 0 $3.0 \mathrm{~min}\left(1.65 \mathrm{~mL} \mathrm{~min}{ }^{-1}\right) ; 3.0-5.0\left(1.65-1.20 \mathrm{~mL} \mathrm{~min}^{-1}\right)$; and $5.0-10.0 \mathrm{~min}\left(1.20 \mathrm{~mL} \mathrm{~min}^{-1}\right)$ [11].

\section{Flow injection procedure}

Figure 1 depicts the merging zones FIA system used to determine tetracycline residues in the egg supplement samples. In the sampling position, the solutions of the standard or sample $(\mathrm{S})$ and the chromogenic reagent $(\mathrm{R})$ filled the SL and RL loops, respectively. In the injection position, discrete volumes of $\mathrm{S}$ and $\mathrm{R}$ were loaded using the carrier solution of $5.0 \times 10^{-2} \mathrm{~mol} \mathrm{~L}^{-1}$ sodium acetate $(\mathrm{C})$ and were mixed at the confluence point $(\mathrm{X})$. The mixture passed through the reaction coil (RC, $300 \mathrm{~cm}$ ), which was kept at $45{ }^{\circ} \mathrm{C}$ (optimized temperature) in a thermostatic bath, and the product formed was carried to the detector flow cell (LWCC). After measurement of the absorbance maximum at $435 \mathrm{~nm}$, the injector-commutator was switched back to the initial position to start another cycle. The detector signal was previously adjusted to zero while pumping the carrier solution at a flow rate of $1.9 \mathrm{~mL} \mathrm{~min}^{-1}$, in the absence of tetracycline antibiotics. Peak height was used as the analytical signal, and its magnitude (absorbance) was proportional to the tetracycline concentration in the sample. SpectraSuite software (Ocean Optics) was used for data acquisition. The proposed flow method provided a frequency of 52 samples per hour.

\section{Statistical analysis}

Multivariate methodologies reduce the number of experiments to be undertaken and improve the quality of information obtained, by means of chemometric analysis of the results, which substantially decreases the duration and cost of the laboratory work required [32]. In this context, a central composite design and a fractional factorial design were used to optimize the FIA and US-DLLME procedures, respectively. The corresponding matrices were developed using Minitab 16 software and optimization graphs were constructed using Statistica 8.0 software.

Data analysis was performed with OriginLab software, using two-way ANOVA tests, and the results were compared using Snedecor's $F$ test and the Student's $t$ test [32]. The confidence interval was set at $95 \%$ in all cases.

\section{Sample preparation}

The real samples used were some protein supplements of high biological value based on albumin coming from egg, which are widely consumed by athletes and sports practitioner, being essential for muscle growth and regeneration. Egg-based protein supplements were obtained from local sports supplement shops in the city of Araraquara (São Paulo State, Brazil) and were stored in a dehumidified environment until analysis. All the samples were tested prior to their expiry dates. Egg supplements free of tetracycline antibiotics were used as blank samples during the validation process. 
For the preparation, about $2.5 \mathrm{~g}$ of egg protein supplement and $20 \mathrm{~mL}$ of deionized water were transferred to a $50-\mathrm{mL}$ polypropylene centrifuge tube, followed by vigorous stirring using a vortex mixer until complete sample dissolution. Fortified samples were prepared by addition of known amounts of analytes. Afterwards, $5 \mathrm{~mL}$ of acetonitrile and $5 \mathrm{~mL}$ of $0.306 \mathrm{~mol} \mathrm{~L}^{-1}$ trichloroacetic acid were added for the protein precipitation, followed by vigorous vortex agitation for $1 \mathrm{~min}$. After centrifuging the sample for $10 \mathrm{~min}$ at $4{ }^{\circ} \mathrm{C}$ and $9000 \mathrm{rpm}$ using a relative centrifugal force of $13,674 \times \mathrm{g}$, the supernatant was used for further analysis, being collected and evaporated to about $4 \mathrm{~mL}$ on a heating plate. Prior to the US-DLLME procedure, the solution that remained was diluted to $5.0 \mathrm{~mL}$ with deionized water and filtered using a hydrophilic Millex-HV PVDF filter (Millipore, $0.45 \mu \mathrm{m}$ ).

\section{Procedure for US-DLLME}

Briefly, the 5-mL egg supplement extract was placed in a $15-\mathrm{mL}$ centrifuge tube, followed by rapid injection of $300 \mu \mathrm{L}$ of ethanol (as disperser solvent) containing $100 \mu \mathrm{L}$ of chloroform (as extraction solvent) using a syringe. The mixture was vortexed for $1 \mathrm{~min}$, until formation of a cloudy solution (which was stable for a long time), and then ultrasonicated for $4.0 \mathrm{~min}$ using an ultrasonic water bath to enhance the extraction of tetracyclines from aqueous solution into the microdroplets of chloroform. The separation was performed by rapid centrifugation using with a $\mathrm{g}$-force of $13,674 \times g$ at $9000 \mathrm{rpm}$ and $4{ }^{\circ} \mathrm{C}$ for $8 \mathrm{~min}$ (a reasonable time for obtaining the best extraction recovery). The dispersed fine particles of extraction solvent containing the analytes were sedimented in the bottom of the centrifuge tube. The upper aqueous phase was removed with a syringe and the settled phase was dried under a flow of nitrogen in a $10-\mathrm{mL}$ volumetric flask. Subsequently, $4 \mathrm{~mL}$ of $5.0 \times 10^{-2} \mathrm{~mol} \mathrm{~L}^{-1}$ sodium acetate was added and the flask was completed with deionized water before analysis using the FIA system.

\section{Method validation}

The proposed US-DLLME-LWCC method was validated using blank extracts of egg supplement samples that did not contain the target tetracyclines. The analytical parameters evaluated included linearity, matrix effects, limits of detection (LOD) and quantification (LOQ), precision, and extraction recovery.

Matrix-matched standard calibration curves were prepared by addition of the four tetracyclines to blank egg supplement extracts at different concentrations $\left(100-400 \mu \mathrm{g} \mathrm{kg}^{-1}\right)$. These solutions were treated according to the same US-DLLME procedure used for the samples after protein precipitation step. The regression equation parameters (slope and intercept) were determined using least squares linear regression analysis. The limits of detection and quantification were calculated according to the IUPAC recommendations [33] as 3 and 10 times, respectively, the standard deviation of measurements of 10 blank solutions, divided by the slope of the analytical curve. Recovery and precision assays were performed over a period of 3 days, with each sample analyzed in triplicate. The recoveries were expressed as percentages, considering the added analyte concentration. The relative standard deviations ( $\% \mathrm{RSD}$ ) were used to describe the variability of the measurements at each concentration level. Taking into account the adopted volumes and concentrations, the correlation factor from microgram per liter to microgram per kilogram was 1:4.

\section{Comparative method}

A reference HPLC-DAD technique was used for comparison with the results obtained by the proposed methodology, as described by Konstantina et al. [11]. After extraction of the tetracyclines from the egg samples, a solid-phase extraction purification procedure was performed. A linear relationship was obtained in the range $0.5-10.0 \mu \mathrm{g} \mathrm{mL}^{-1}$. No previous reports concerning the determination of tetracycline antibiotics in egg supplements were found in the literature, so a reference method for the determination of tetracycline antibiotics residues in chicken muscle and eggs was applied in egg supplement samples without any modifications in the sample preparation procedure described therein, which allowed its use as a comparative technique.

\section{Results and discussion}

\section{FIA system optimization}

The significant parameters for the merging zones FIA system with LWCC were as follows: reaction coil length, temperature, sample loop size, reagent loop size, flow rate, and the concentrations of $\mathrm{p}$-sulfanilic acid $\left(\mathrm{C}_{6} \mathrm{H}_{7} \mathrm{NO}_{3} \mathrm{~S}\right)$, hydrochloric acid $(\mathrm{HCl})$, sodium nitrite $\left(\mathrm{NaNO}_{2}\right)$, and sodium acetate $\left(\mathrm{NaC}_{2} \mathrm{H}_{3} \mathrm{O}_{2}\right)$. Preliminary tests were used to establish the optimal values for the chemical variables involved in the colorimetric reaction $\left(\mathrm{C}_{6} \mathrm{H}_{7} \mathrm{NO}_{3} \mathrm{~S}, \mathrm{HCl}, \mathrm{NaNO}_{2}\right.$, and $\mathrm{NaC}_{2} \mathrm{H}_{3} \mathrm{O}_{2}$ concentrations of $1.2 \times 10^{-4}, 2.0 \times 10^{-4}, 2.4 \times 10^{-4}$, and $5.0 \times 10^{-2} \mathrm{~mol} \mathrm{~L}^{-1}$, respectively), as well as the sizes of the sample and reagent loops (402 and $603 \mu \mathrm{L}$, respectively). Additionally, investigation was made of the effects of temperature, reaction coil length, and flow rate.

\section{Effect of temperature}

In previous tests, it was found that use of an LWCC-FIA system with merging zones (Fig. 1) resulted in arrival of the samples at the detector before complete formation of the 
desired azo compounds. This could have been due to low reactant concentrations, which slowed the rate of the reaction, and the resulting sensitivity was not sufficient for determination of the tetracycline residues using their azo derivatives. Nonetheless, new tests were performed in order to improve the intensity of the analytical signal.

The influence of the reaction coil temperature was investigated by varying the temperature from 26 to $55^{\circ} \mathrm{C}$ using a thermostatic bath, with all experiments performed in quintuplicate. The results obtained are shown in Fig. 2. There was a significant increase of peak height (absorbance) as the temperature was increased, with a maximum response reached at $45^{\circ} \mathrm{C}$, followed by a gradual decrease of the signal with further heating, probably due to degradation of the product formed. The reaction blank was not affected by the temperature, indicating that the increase in absorbance was associated with the desired product, rather than spectral interferences. The temperature influence on the reaction sensitivity was equally observed for all studied tetracyclines. A temperature of $45^{\circ} \mathrm{C}$ was therefore selected in the subsequent experiments.

Statistical evaluation of the experimental data by means of ANOVA tests revealed no significant differences between the reaction blanks measured at different temperatures ( $95 \%$ confidence level, $P=0.925, F=0.301)$. However, in the case of the reaction products (tetracycline azo derivatives), heating of the reaction coil had a significant effect $(P<0.0001$, $F=2647$ ).

The significant effect of temperature on the diazo coupling reaction was probably due to the greater movement of molecules when the reaction coil was heated, which favored successful collisions with sufficient activation energy to break the existing bonds and form new bonds [34]. As a result, the reaction rate increased and the products were formed in a shorter time.

Although the sensitivity of the reaction was improved by a modest increase of temperature, the reaction rate was also influenced by other physical parameters of the system such as the reaction coil length and the flow rate. It was therefore necessary to perform a study to investigate the effects of these parameters, at the optimized temperature.



Fig. 2 Temperature effect on reaction sensitivity

\section{Central composite design}

The best analytical conditions, considering the reaction coil length and the flow rate, with in-line heating, were obtained by multivariate analysis using a central composite design and two-dimensional response surface analysis [32]. The effects of these variables on the absorbance signal were studied using five levels $(-\sqrt{2},-1,0,1$, and $\sqrt{2})$. The points (levels) of a central composite design are $\sqrt{2}$ coded units distant from the central point (coded as zero point), so all the points lie on a circumference with radius $\sqrt{2}$. The encoding of the variables is shown in the Electronic Supplementary Material (ESM), Table S1. All the optimization experiments using LWCC spectrophotometric detection were performed at $45{ }^{\circ} \mathrm{C}$, and the tetracycline concentration was kept constant at $400 \mu \mathrm{g} \mathrm{L}^{-1}$.

The resulting response surface and the corresponding contour curves are shown in ESM Fig. S1 as a function of reaction coil length and flow rate onto the detection absorbance. The darker region within the response surface represents the maximum absorbance obtained under optimum reaction conditions, indicating the levels of the factors at which the analysis was most sensitive.

The quadratic regression model could be described by:

$A=0.226+0.003 x-6.033 \times 10^{-6} x^{2}-0.102 y-0.015 y^{2}+$ $3.056 \times 10^{-4} x y$,

where $A$ is the response factor corresponding to the absorbance value, $x$ is the flow rate, and $y$ is the reaction coil length. The calculated regression coefficient $\left(r^{2}\right)$ was $0.96 \%$, indicating that the equation obtained was able to explain the relationship between the experimental results and the effects of the factors studied.

Analysis of the surface graph and its projection (Fig. S1, ESM) enabled identification of the optimal region for tetracyclines detection, showing that points below the $-\sqrt{2}$ level should be used for the flow rate. However, this level $(-\sqrt{2})$ was selected considering the minimum flow rate required to sustain a continuous flow in the FIA system. The reaction coil length was successfully optimized at the 0 level. The maximum absorbance value (0.593) was achieved using a flow rate of $1.9 \mathrm{~mL} \mathrm{~min}^{-1}$ and a reaction coil length of $3.0 \mathrm{~m}$, corresponding to levels $-\sqrt{2}$ and 0 , respectively. The optimized values of the variables enabled uniform peaks and a stable baseline to be achieved during absorbance measurements at $435 \mathrm{~nm}$.

\section{Optimization of the US-DLLME procedure}

The optimum experimental conditions for the quantitative extraction of tetracycline drugs by US-DLLME were established considering the parameters that significantly affected the extraction efficiency. These included the types and volumes of the extraction and disperser solvents, and the times used for 
sonication, extraction, and centrifugation. The optimization was performed using $5 \mathrm{~mL}$ of blank egg supplement extract that had been previously spiked with the analytes at concentrations of $200 \mu \mathrm{g} \mathrm{kg}^{-1}$. The performance of the methodology was characterized in terms of the extraction recovery ( $\mathrm{R} \%)$, calculated using the ratio between the amount of analyte extracted in the sedimented phase and the total spiked amount. The results were obtained as the mean values of three extractions.

\section{Selection of the extraction and disperser solvents}

The selection of appropriate extraction and disperser solvents for use in a DLLME method is critical for achieving satisfactory analyte recovery. The extraction solvent has to satisfy several requirements including low solubility in water, good ability to extract the target analytes, higher density than water, miscibility with the disperser solvent, and suitability for use with the selected analytical technique [23]. In selection of the disperser solvent, it is necessary to ensure its miscibility in both water (the sample) and the extraction solvent (the organic phase) [23].

Based on the above considerations, the extraction of the tetracyclines was evaluated using different combinations of dichloromethane $\left(\mathrm{CH}_{2} \mathrm{Cl}_{2}, 1.33 \mathrm{~g} \mathrm{~cm}^{-3}\right.$ density), chloroform $\left(\mathrm{CHCl}_{3}, 1.49 \mathrm{~g} \mathrm{~cm}^{-3}\right.$ density), and carbon tetrachloride $\left(\mathrm{CCl}_{4}\right.$, $1.58 \mathrm{~g} \mathrm{~cm}^{-3}$ density) as the extraction solvent. The disperser solvents tested were methanol $\left(\mathrm{CH}_{3} \mathrm{OH}\right)$, ethanol $\left(\mathrm{C}_{2} \mathrm{H}_{5} \mathrm{OH}\right)$, acetone $\left(\mathrm{C}_{2} \mathrm{H}_{6} \mathrm{CO}\right)$, and acetonitrile $\left(\mathrm{CH}_{3} \mathrm{CN}\right)$.

First, the effect of the type of extraction solvent was evaluated using $500 \mu \mathrm{L}$ of acetonitrile as the disperser solvent and $200 \mu \mathrm{L}$ of each extraction solvent. Several different combinations of chloroform with each disperser solvent were evaluated using the same volume ratio.

The effects of the different extraction solvents on extraction performance are illustrated in Fig. 3. It can be seen that the

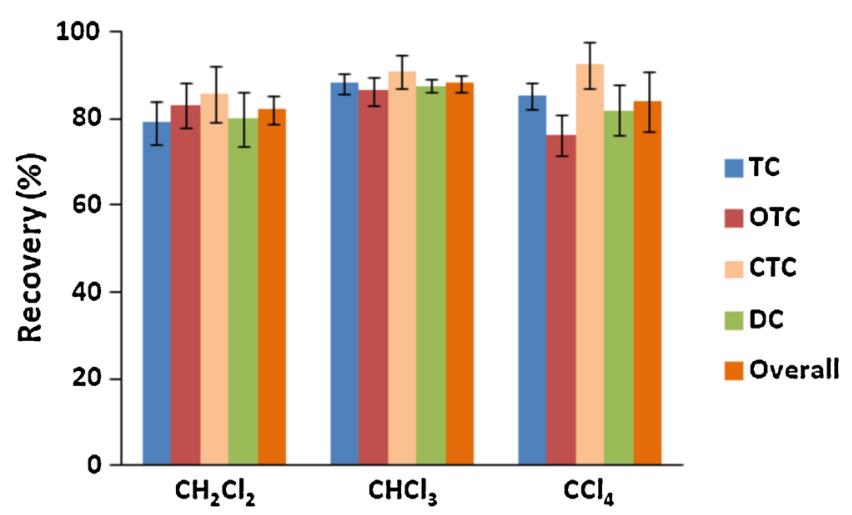

Fig. 3 Effects of different extraction solvents on tetracycline recoveries. Extraction conditions: $5 \mathrm{~mL}$ of blank egg supplement extract; spiked concentration of $200 \mu \mathrm{g} \mathrm{kg}^{-1} ; 200 \mu \mathrm{L}$ of extraction solvent; $500 \mu \mathrm{L}$ of acetonitrile disperser solvent; $10 \mathrm{~min}$ extraction time; $8 \mathrm{~min}$ centrifugation time most efficient extraction was obtained for chlortetracycline (85.7-92.3\%), probably due to the higher affinity of this analyte for the selected chlorinated solvents. The use of dichloromethane (79.0-85.7\%) and carbon tetrachloride (76.2$92.3 \%$ ) provided satisfactory recoveries, although the results were quite variable. Chloroform was most effective for extraction of the individual tetracyclines, with recoveries in the range $86.3-90.7 \%$ and the highest overall recovery of $88.1 \%$. Hence, chloroform was selected as the extraction solvent.

The recoveries for extraction of the tetracyclines using methanol, ethanol, acetone, and acetonitrile as disperser solvents were in the ranges 74.0-85.3, 86.2-91.4, 62.5-74.3, and 84.4-93.2\%, respectively (Fig. 4). There was poor phase separation when acetone was used as the disperser solvent, which affected the volume of the settled organic phase and led to an overall recovery below $70 \%$. The best overall recoveries were obtained with ethanol $(89.2 \%)$ and acetonitrile $(88.6 \%)$, which could be attributed to better dispersion of the extraction solvent (chloroform) in the aqueous phase. Song et al. [7] reported similar results to those obtained here with acetonitrile, but using the ionic liquid 1-butyl-3methylimidazolium hexafluorophosphate $\left(\left[\mathrm{C}_{4} \mathrm{MIM}\right]\left[\mathrm{PF}_{6}\right]\right)$ for the determination of tetracyclines in eggs by HPLC [7]. Nonetheless, ethanol was selected as the disperser solvent in the subsequent studies because it resulted in the smallest standard deviation (SD) values, indicative of good precision. Furthermore, ethanol is a greener solvent and is inexpensive.

\section{Fractional factorial design for US-DLLME parameters}

The effects of the analytical parameters on the US-DLLME extraction of the tetracyclines from the egg supplement samples were studied using a $2^{5-2}$ fractional factorial design [32]. The variables considered here were the volume of extraction solvent, volume of disperser solvent, sonication time,



Fig. 4 Effects of different disperser solvents on tetracycline recoveries. Extraction conditions: $5 \mathrm{~mL}$ of blank egg supplement extract; spiked concentration of $200 \mu \mathrm{g} \mathrm{kg}^{-1} ; 200 \mathrm{~mL}$ of chloroform extraction solvent; $500 \mathrm{~mL}$ of disperser solvent; $10 \mathrm{~min}$ extraction time; $8 \mathrm{~min}$ centrifugation time 
extraction time, and centrifugation time. In DLLME, the extraction time is defined as the time interval elapsed between the injection of the solvent mixture and the start of the centrifugation $[7,23,24]$.

Two levels (low and high) were selected for each parameter, according to tests performed previously. The variables and their levels are provided in Table 1. Eight experiments were carried out randomly, in triplicate, using a constant $200 \mu \mathrm{g} \mathrm{kg}^{-1}$ concentration of the tetracyclines.

The results showed that the low level of ethanol was sufficient for effective dispersion of the extraction solvent (chloroform) at high or low levels. There was complete formation of a cloudy suspension of microdrops, which minimized the solubility of the tetracyclines in the aqueous phase and improved the recoveries, due to an increase in the distribution coefficient. This behavior was assisted by the use of sonication immediately after injection of the solvent mixture.

The extraction efficiencies obtained with and without sonication were in the ranges $92-95$ and 86-89\%, respectively, so sonication resulted in a gain in recovery of about $6 \%$. Sonication promoted the formation of a cloudier solution, leading to rapid mass transfer of the analytes from the aqueous solution (sample extract), regardless of the extraction solvent viscosity. Therefore, the contact surface between the extraction solvent droplets and the aqueous sample was considerably enlarged, resulting in a shorter equilibration time. These observations confirmed the important role of sonication in the extraction.

Figure 5 illustrates the Pareto diagram obtained from fitting the experimental data, with the effects of the variables presented in the form of bars, where the length of the bar is proportional to the absolute value of the associated regression coefficient or estimated effect. The effects of all the parameters and interactions were standardized by dividing each effect by its standard error. The order in which the bars are displayed corresponds to the order of the magnitude of the effect of each variable. The Pareto chart also includes a discontinuous



Fig. 5 Pareto chart showing the effects of the selected variables, using a $2^{5-2}$ fractional factorial design. Other extraction conditions: $5 \mathrm{~mL}$ of blank egg supplement extract; spiked concentration of $200 \mu \mathrm{g} \mathrm{kg}^{-1}$; chloroform extraction solvent; ethanol disperser solvent

vertical line that indicates the statistical significance limit $(P=0.05)$. An effect was considered significant when the corresponding bar crossed this vertical line.

The most significant effect was associated with the centrifugation time, with the best response obtained using the high $(+1)$ level (Fig. 5). Other less significant variables were adjusted according to the experimental results, with the maximum $(+1)$ level for sonication time and the minimum $(-1)$ levels for the solvents (extraction and disperser) and extraction time. Although the sonication time did not have a significant effect, the use of sonication was fundamental for the development of this new procedure. Recovery of the tetracyclines was not significantly affected by the extraction time, with the first 5 min being sufficient to reach equilibrium. Importantly, it was possible to select the minimum values for the disperser and extraction solvents, due to the use of ultrasound in the extraction. The absolute values corresponding to the selected levels of the parameters are shown in the Pareto graph (Fig. 5).

The optimized values of the variables investigated, which provided the best tetracycline recoveries, were as follows:
Table 1 Coded and uncoded levels for variables of the fractional factorial design $\left(2^{5-2}\right)$

\begin{tabular}{llllll}
\hline Expt. & Factors $^{\mathrm{a}}$ & & & & \\
\cline { 2 - 6 } & $\begin{array}{l}\text { Extraction } \\
\text { solvent }(\mu \mathrm{L})\end{array}$ & $\begin{array}{l}\text { Disperser } \\
\text { solvent }(\mu \mathrm{L})\end{array}$ & $\begin{array}{l}\text { Sonication time } \\
(\mathrm{min})\end{array}$ & $\begin{array}{l}\text { Extraction time } \\
(\mathrm{min})\end{array}$ & $\begin{array}{l}\text { Centrifugation time } \\
(\mathrm{min})\end{array}$ \\
\hline 1 & $100(-1)$ & $300(-1)$ & $0(-1)$ & $10(+1)$ & $8(+1)$ \\
2 & $200(+1)$ & $300(-1)$ & $4(+1)$ & $5(-1)$ & $8(+1)$ \\
3 & $200(+1)$ & $500(+1)$ & $0(-1)$ & $10(+1)$ & $3(-1)$ \\
4 & $200(+1)$ & $300(-1)$ & $0(-1)$ & $5(-1)$ & $3(-1)$ \\
5 & $100(-1)$ & $300(-1)$ & $4(+1)$ & $5(-1)$ & $8(+1)$ \\
6 & $100(-1)$ & $500(+1)$ & $0(-1)$ & $10(+1)$ & $3(-1)$ \\
7 & $100(-1)$ & $500(+1)$ & $4(+1)$ & $5(-1)$ & $8(+1)$ \\
8 & $200(+1)$ & $500(+1)$ & $4(+1)$ & $10(+1)$ & $3(-1)$ \\
\hline
\end{tabular}

${ }^{\text {a }}$ Coded values are shown in parentheses 
$100 \mu \mathrm{L}$ of extraction solvent, $300 \mu \mathrm{L}$ of disperser solvent, and 4,5 , and $8 \mathrm{~min}$ for the sonication, extraction, and centrifugation times, respectively. Optimization of the significant parameters improved the performance of the proposed US-DLLME method and minimized the consumption of organic solvents, hence contributing to the goals of green analytical chemistry.

\section{Analytical features of the US-DLLME-FIA method}

The effects on the colorimetric reaction employed here of the most common undesirable substances found in animalderived foods, such as sulfonamides, aminoglycosides, macrolides, amphenicols, and beta-lactams, have been investigated in previous studies. The effects were not considered significant, since the changes in the tetracycline signal did not exceed $5 \%$ [31]. Here, no interference in the reaction was observed with heating, indicating good selectivity of the proposed method with LWCC-based spectrophotometric detection.

The figures of merit for the method, determined under the optimal conditions, are summarized in Table 2. The calibration curves for the four tetracyclines showed good linearity in the concentration range $30-600 \mu \mathrm{g} \mathrm{L}^{-1}$, with correlation coefficients $\left(r^{2}\right)$ greater than 0.9974 . The LODs and LOQs were in the ranges 6.4-11.1 and 21.3-37.0 $\mu \mathrm{g} \mathrm{L}^{-1}$, respectively. These values were satisfactory considering the maximum permissible levels of tetracycline contaminants in eggs established in the Canadian $\left(400 \mu \mathrm{g} \mathrm{kg}^{-1}\right)$ [8], EU $\left(200 \mu \mathrm{g} \mathrm{kg}^{-1}\right)$ [10], and Codex Alimentarius $\left(400 \mu \mathrm{g} \mathrm{kg}^{-1}\right)$ [9] regulations. The results indicated that the US-DLLMEFIA method developed here was sufficiently sensitive for the determination of tetracycline residues in egg supplement samples.

Possible alteration of the analytical signal due to matrix effects was evaluated using $t$ tests to compare the slopes of calibration graphs constructed using standard solutions of each tetracycline with those of graphs obtained by spiking the matrix with same standard solutions [24]. The calculated $t$ values ranged between 1.732 and 3.464, not exceeding therefore the critical value $(t=4.303)$, which indicates that there were no statistically significant differences among the slopes

Table 2 Figures of merit for the US-DLLME-FIA method

\begin{tabular}{lllll}
\hline Analyte & TC & OTC & CTC & DC \\
\hline $\begin{array}{l}\text { Linear range } \\
\left(\mu \mathrm{g} \mathrm{L}^{-1}\right)\end{array}$ & $30-600$ & $30-600$ & $30-600$ & $30-600$ \\
$r^{2}$ & 0.9992 & 0.9991 & 0.9974 & 0.9983 \\
Slope $\left(\times 10^{-3}\right)$ & 1.22 & 1.18 & 1.06 & 1.03 \\
Intercept $\left(\times 10^{-2}\right)$ & -1.58 & -1.75 & -1.02 & -0.36 \\
LOD $\left(\mu \mathrm{g} \mathrm{L}^{-1}\right)$ & 6.4 & 7.8 & 9.7 & 11.1 \\
LOQ $\left(\mu \mathrm{g} \mathrm{L}^{-1}\right)$ & 21.3 & 26.1 & 32.4 & 37.0 \\
\hline
\end{tabular}

at the $95 \%$ confidence level and that matrix effects were absent.

Table 3 shows the intra-day and inter-day accuracies and precisions obtained for the fortified egg supplement samples. The intra-day and inter-day average recoveries of the tetracyclines were in the ranges 85.7-95.8 and 88.2-96.4\%, respectively, and the intra-day and inter-day RSD values were in the ranges 2.5-9.6 and 3.2-9.8\%, respectively. According to the Codex Alimentarius criteria [35], these values are within the acceptable ranges for intermediate precision $(\leq 15 \%$ RSD) and recovery $(70-110 \%)$. The data indicated that the compositions of the egg-based protein supplements analyzed did not significantly interfere in the determination of tetracyclines at trace levels. The methodology can therefore be used to determine this class of antimicrobials with adequate precision and accuracy, helping to protect consumers from exposure to tetracycline residues in egg-based protein supplements.

\section{Application to egg supplement samples}

Antibiotics residues have frequently been found in monitoring programs of animal-derived foods [5-7, 13, 16, 17]. The new US-DLLME-LWCC method was applied using seven eggbased protein supplement samples. None of the samples showed the presence of tetracyclines at concentrations exceeding the detection limits $\left(6.4-11.1 \mu \mathrm{g} \mathrm{L}^{-1}\right)$. The samples therefore complied with the MRL values $\left(200-400 \mu \mathrm{g} \mathrm{kg}^{-1}\right)$ [8-10], confirming that the egg supplements were suitable for human consumption.

For comparison purposes, the samples (spiked and not spiked) were also analyzed by a reference HPLC method [11]. The results (Table 4) were compared using $F$ tests and $t$ tests at a $95 \%$ confidence level. The calculated $F$ and $t$ values did not exceed the critical values, showing that there were no significant differences between the results provided by the proposed (US-DLLME-FIA) and comparative (SPE-HPLC) methods.

The US-DLLME extraction procedure was easily integrated to a flow injection system equipped with an LWCC-based spectrophotometric detector and an in-line controlled heating system. Under optimum conditions, 52 samples per hour could be analyzed by the proposed method, whereas the analytical frequency of the reference HPLC method [11] was much slower, due to a chromatographic run time of $10 \mathrm{~min}$. The FIA system employed offers the possibility of automation, better precision, operational simplicity, low analytical costs, and low consumption of reagents (about $12.1 \mu \mathrm{g}$ of $\mathrm{p}$ sulfanilic acid, $10.0 \mu \mathrm{g}$ of sodium nitrite, and $12.3 \mathrm{mg}$ of sodium acetate) and samples $(402 \mu \mathrm{L})$. In contrast, the comparative technique requires large volumes of organic solvents that can be harmful to health and the environment [21]. Furthermore, HPLC analysis incurs additional costs associated with the specialist technical support required. 
Table 3 Intra-day and inter-day accuracies and precisions for the spiked egg supplement samples

\begin{tabular}{|c|c|c|c|c|c|}
\hline \multirow[t]{2}{*}{ Analyte } & \multirow[t]{2}{*}{ Added amount $\left(\mu \mathrm{g} \mathrm{kg}^{-1}\right)$} & \multicolumn{2}{|c|}{ Intra-day $(n=3)$} & \multicolumn{2}{|c|}{ Inter-day $(n=9)$} \\
\hline & & Recovery (\%) & RSD (\%) & Recovery (\%) & $\operatorname{RSD}(\%)$ \\
\hline \multirow[t]{4}{*}{$\mathrm{TC}$} & 100 & 85.7 & 8.0 & 88.5 & 6.4 \\
\hline & 200 & 94.1 & 4.4 & 91.0 & 7.2 \\
\hline & 300 & 90.5 & 3.6 & 90.3 & 4.8 \\
\hline & 400 & 93.8 & 6.1 & 94.1 & 5.3 \\
\hline \multirow[t]{4}{*}{ OTC } & 100 & 88.8 & 9.6 & 88.2 & 8.7 \\
\hline & 200 & 90.2 & 7.1 & 91.0 & 5.5 \\
\hline & 300 & 92.1 & 2.5 & 93.4 & 3.2 \\
\hline & 400 & 93.7 & 8.3 & 94.2 & 6.1 \\
\hline \multirow[t]{4}{*}{ CTC } & 100 & 92.5 & 6.2 & 89.3 & 9.2 \\
\hline & 200 & 94.0 & 5.0 & 92.8 & 6.4 \\
\hline & 300 & 95.8 & 3.5 & 93.5 & 7.3 \\
\hline & 400 & 93.2 & 4.6 & 96.4 & 5.2 \\
\hline \multirow[t]{4}{*}{ DC } & 100 & 86.6 & 9.3 & 89.2 & 9.8 \\
\hline & 200 & 88.0 & 5.6 & 90.6 & 7.7 \\
\hline & 300 & 89.3 & 7.4 & 91.4 & 6.3 \\
\hline & 400 & 91.6 & 8.0 & 92.7 & 7.2 \\
\hline
\end{tabular}

In the reference method, sample extraction was performed using SPE [11], which is generally a tedious and timeconsuming procedure. In addition, the quantities of organic solvents required are much greater than in microextraction methods [22], and the SPE cartridges can be expensive. In contrast, the US-DLLME procedure provides a simple, rapid, inexpensive, and efficient extraction of tetracyclines from egg supplement samples. The use of toxic solvents is considerably reduced (about $0.1 \mathrm{~mL}$ of chloroform is used as the extraction solvent), and there is the ability to use greener solvents (about $0.3 \mathrm{~mL}$ ethanol is used as the disperser solvent).

Implementation of new methodologies that enable elimination or reduction of toxic solvents used in analytical activities is essential in order to protect human health and the environment [21]. The US-DLLME-FIA method developed here is environmental friendly and contributes to such a goal.

\section{Conclusions}

A novel method using US-DLLME, followed by spectrophotometric FIA using a liquid waveguide capillary cell, was developed for the extraction and determination of tetracycline compounds in egg-based protein supplements. The sensitivity of the method was substantially enhanced (5.12-fold) by including a heating step during analyses. After optimization, US-DLLME provided cleaner extractions compared to traditional extraction methods and greatly reduced the consumption of toxic solvents, due to the miniaturized extraction procedure. The new US-DLLME-FIA method is an analytical tool that is environmentally friendly and conforms to the principles of green chemistry. It is simpler, faster, cheaper, and more efficient, compared to other methodologies described in the literature. This is the first report describing a US-
Table 4 Determination of tetracycline antibiotics in egg supplement samples

\begin{tabular}{lllllll}
\hline Sample & Tetracycline added & $\begin{array}{l}\text { Added amount } \\
\left(\mu \mathrm{kg}^{-1}\right)\end{array}$ & $\begin{array}{l}\text { US-DLLME-FIA } \\
\text { method }^{\mathrm{a}}\end{array}$ & $\begin{array}{l}\text { SPE-HPLC } \\
\text { method }^{\mathrm{a}}\end{array}$ & $T$ test $^{\mathrm{b}}$ & $F$ test $^{\mathrm{c}}$ \\
\hline 1 & None & 0 & - & - & - & - \\
2 & None & 0 & - & - & - & - \\
3 & None & 0 & - & - & - & - \\
4 & TC & 200 & $187 \pm 7$ & $179 \pm 4$ & 1.806 & 3.32 \\
5 & OTC & 400 & $380 \pm 8$ & $382 \pm 14$ & 0.219 & 2.58 \\
6 & CTC & 200 & $194 \pm 9$ & $197 \pm 8$ & 0.525 & 1.22 \\
7 & DC & 100 & $092 \pm 10$ & $085 \pm 12$ & 0.747 & 1.39 \\
\hline
\end{tabular}

${ }^{a}$ Average of three determinations $(n=3) \pm$ the corresponding standard deviation (SD)

${ }^{\mathrm{b}}$ Student's $t$ test tabulated value $=2.776(95 \%$ confidence level $)$

${ }^{\text {c }}$ Snedecor's $F$ test tabulated value $=19.00$ (95\% confidence level) 
DLLME technique for the extraction of tetracycline drugs prior to flow injection spectrophotometric analysis based on an LWCC. The integrating of these procedures provided reliable performance and sufficient sensitivity to determine tetracycline residues in egg supplement samples for the purpose of consumer protection.

Acknowledgments The authors would like to thank CNPq for financial support, and the PAEDEX/AUIP/UNESP program for the awarding of a scholarship to M. P. Rodríguez.

\section{Compliance with ethical standards}

Conflict of interest The authors declare that they have no conflicts of interest.

\section{References}

1. Bruno F, Curini R, Di-Corcia A, Nazzari M, Pallagrosi M. An original approach to determining traces of tetracycline antibiotics in milk and eggs by solid-phase extraction and liquid chromatography/mass spectrometry. Rapid Commun Mass Spectrom. 2002;16:1365-76.

2. Dasenaki ME, Thomaidis NS. Multi-residue determination of 115 veterinary drugs and pharmaceutical residues in milk powder, butter, fish tissue and eggs using liquid chromatography-tandem mass spectrometry. Anal Chim Acta. 2015;880:103-21.

3. Sczesny S, Nau H, Hamscher G. Residue analysis of tetracyclines and their metabolites in eggs and in the environment by HPLC coupled with a microbiological assay and tandem mass spectrometry. J Agric Food Chem. 2003;51:697-703.

4. Piatkowska M, Jedziniak P, Zmudzki J. Comparison of different sample preparation procedures for multiclass determination of selected veterinary drug, coccidiostat and insecticide residues in eggs by liquid chromatography-tandem mass spectrometry. Anal Methods. 2014;6:3034 44.

5. Frenich AG, Aguilera-Luiz M, Vidal JLM, Romero-González R. Comparison of several extraction techniques for multiclass analysis of veterinary drugs in eggs using ultra-high pressure liquid chromatography-tandem mass spectrometry. Anal Chim Acta. 2010;661: $150-60$.

6. Jiménez V, Rubies A, Centrich F, Companyó R, Guiteras J. Development and validation of a multiclass method for the analysis of antibiotic residues in eggs by liquid chromatography-tandem mass spectrometry. J Chromatogr A. 2011;1218:1443-51.

7. Song J, Zhang ZH, Zhang YQ, Feng C, Wang GN, Wang JP. Ionic liquid dispersive liquid-liquid microextraction combined with high performance liquid chromatography for determination of tetracycline drugs in eggs. Anal Methods. 2014;6:6459-66.

8. Canada, Food and Drugs Act SOR-87. List of maximum residue limits (MRLs) for veterinary drugs in foods. Canada Gazette Part II. 2013;147:1306.

9. Codex Alimentarius CAC/MRL-2. Maximum residues limits (MRLs) and risk management recommendations (RMRs) for residues of veterinary drugs in Foods. 2014:8.

10. Union E. Commission regulation No 37. On pharmacologically active substances and their classification regarding maximum residue limits in foodstuffs of animal origin. Off $\mathrm{J}$ Eur Union. 2010;L15:19-64.
11. Nikolaidou KI, Samanidou VF, Papadoyannis IN. Development and validation of an HPLC method for the determination of seven tetracycline antibiotics residues in chicken muscle and egg yolk according to 2002/657/EC. J Liq Chrom Relat Tech. 2008;31: 2141-58.

12. Liu Y, Yang H, Yang S, Hu Q, Cheng H, Liu H, et al. Highperformance liquid chromatography using pressurized liquid extraction for the determination of seven tetracyclines in egg, fish and shrimp. J Chromatogr B. 2013;917-918:11-7.

13. Jing T, Niu J, Xia H, Dai Q, Zheng H, Hao Q, et al. Online coupling of molecularly imprinted solid-phase extraction to HPLC for determination of trace tetracycline antibiotic residues in egg samples. J Sep Sci. 2001;34:1469-76.

14. Zurhelle G, Muller-Seitz E, Petz M. Automated residue analysis of tetracyclines and their metabolites in whole egg, egg white, egg yolk and hen's plasma utilizing a modified ASTED system. J Chromatogr B. 2000;739:191-203.

15. Heller DN, Nochetto CB, Rummel NG, Thomas MH. Development of multiclass methods for drug residues in eggs: hydrophilic solidphase extraction cleanup and liquid chromatography/tandem mass spectrometry analysis of tetracycline, fluoroquinolone, sulfonamide, and beta-lactam residues. J Agric Food Chem. 2006;54: 5267-78.

16. Capriotti AL, Cavaliere C, Piovesana S, Samperi R, Laganà A. Multiclass screening method based on solvent extraction and liquid chromatography-tandem mass spectrometry for the determination of antimicrobials and mycotoxins in egg. J Chromatogr A. 2012;1268:84-90.

17. Piatkowska M, Jedziniak P, Zmudzki J. Multiresidue method for the simultaneous determination of veterinary medicinal products, feed additives and illegal dyes in eggs using liquid chromatographytandem mass spectrometry. Food Chem. 2016;197:571-80.

18. Chen L, Liu J, Zeng Q, Wang H, Yu A, Zhang H, et al. Preparation of magnetic molecularly imprinted polymer for the separation of tetracycline antibiotics from egg and tissue samples. J Chromatogr A. 2009;1216:3710-9.

19. Jia X, Shao B, Wu Y, Yang Y, Zhang J. Simultaneous determination of tetracyclines and quinolones antibiotics in egg by ultraperformance liquid chromatography-electrospray tandem mass spectrometry. J AOAC Int. 2008;91:461-8.

20. Jing $\mathrm{T}$, Gao XD, Wang $\mathrm{P}$, Wang $\mathrm{Y}$, Lin $\mathrm{YF}, \mathrm{Hu} \mathrm{XZ}$, et al. Determination of trace tetracycline antibiotics in foodstuffs by liquid chromatography-tandem mass spectrometry coupled with selective molecular-imprinted solid-phase extraction. Anal Bioanal Chem. 2009;393:2009-18.

21. Gałuszka A, Migaszewski Z, Namiesnik J. The 12 principles of green analytical chemistry and the SIGNIFICANCE mnemonic of green analytical practices. Trends Anal Chem. 2013;50:78-84.

22. Saraji M, Boroujeni MK. Recent developments in dispersive liquid-liquid microextraction. Anal Bioanal Chem. 2014;406:202766.

23. Saraji M, Marzban M. Determination of 11 priority pollutant phenols in wastewater using dispersive liquid-liquid microextraction followed by high-performance liquid chromatography-diode-array detection. Anal Bioanal Chem. 2010;396:2685-93.

24. Vázquez MMP, Vázquez PP, Galera MM, García MDG, Uclés A. Ultrasound-assisted ionic liquid dispersive liquid-liquid microextraction coupled with liquid chromatography-quadrupolelinear ion trap-mass spectrometry for simultaneous analysis of pharmaceuticals in wastewaters. J Chromatogr A. 2013;1291:19-26.

25. Farajzadeh MA, Bahram M, Vardast MR, Bamorowat M. Dispersive liquid-liquid microextraction for the analysis of three organophosphorus pesticides in real samples by high performance liquid chromatography-ultraviolet detection and its optimization by experimental design. Microchim Acta. 2011;172:465-70. 
26. Diao C, Li C, Yang X, Sun A, Liu R. Magnetic matrix solid phase dispersion assisted dispersive liquid liquid microextraction of ultra trace polychlorinated biphenyls in water prior to GC-ECD determination. Microchim Acta. 2016;183:1261-8.

27. Kozani RR, Mofid-Nakhaei J, Jamali MR. Rapid spectrophotometric determination of trace amounts of palladium in water samples after dispersive liquid-liquid microextraction. Environ Monit Assess. 2013;185:6531-7.

28. Bidari A, Hemmatkhah P, Jafarvand S, Hosseini MRM, Assadi Y. Selenium analysis in water samples by dispersive liquid-liquid microextraction based on piazselenol formation and GC-ECD. Microchim Acta. 2008;163:243-9.

29. Jafarvand S, Shemirani F. Supramolecular-based dispersive liquidliquid microextraction: a novel sample preparation technique for determination of inorganic species. Microchim Acta. 2011;173: $353-9$.
30. Frugeri PM, Lago AC, Wisniewski C, Luccas PO. A spectrophotometric flow injection system for streptomycin determination in veterinary samples. Spectrochim Acta A. 2014;117:304-8.

31. Rodríguez MP, Pezza HR, Pezza L. Simple and clean determination of tetracyclines by flow injection analysis. Spectrochim Acta A. 2016;153:386-92.

32. Mason RL, Gunst RF, Hess JL. Statistical design and analysis of experiments. 2nd ed. Hoboken, New Jersey: Wiley; 2003.

33. Currie L, Horwitz W. IUPAC recommendations for defining and measuring detection and quantification limits. Anal Magazine. 1994;22:M24-6.

34. Atkins P, Jones L. Chemical principles: questioning the modern life and environment. 5th ed. Bookman; 2011.

35. Codex Alimentarius CAC/GL-71. Guidelines for the design and implementation of national regulatory food safety assurance programme associated with the use of veterinary drugs in food producing animals. 2009:22. 